\title{
Management of Multiple Myeloma
}

\author{
Presented by Shaji K. Kumar, MD
}

\section{Abstract}

The most recent NCCN Guidelines for Multiple Myeloma include a ranking of the many treatment options for various settings as "preferred," "other," and "useful in certain circumstances." For patients eligible for autologous stem cell transplant (ASCT), the preferred regimen remains bortezomib/lenalidomide/dexamethasone (category 1) or bortezomib/cyclophosphamide/dexamethasone. Upfront ASCT also remains a preferred strategy for patients who are transplant-eligible, despite highly effective newer agents such as induction therapy. Double (tandem) ASCT may benefit patients with high-risk cytogenetics, such as $17 p$ deletion. Lenalidomide maintenance is the standard posttransplant approach and results in improved progression-free and overall survivals. For relapsed disease, a host of new agents have been shown to improve outcomes, mostly in combination with bortezomib or lenalidomide, but their selection depends largely on response and tolerability to prior therapies.

\section{Treatment Options for Myeloma Now Categorized}

The predominant change to the NCCN Guidelines for Multiple Myeloma (MM) is the grouping of treatment regimens according to "preferred," "other," and "useful in certain circumstances," according to Shaji K. Kumar, MD, Professor of Medicine, Mayo Clinic Cancer Center, and Panel Chair of the NCCN Guidelines for MM, who described MM management at the NCCN 23rd Annual Conference. Although these categories already exist within other NCCN Guidelines, this is the first version of the guidelines that has incorporated this ranking. "This gives a little more guidance in terms of which regimen to use, especially now that we have so many different combinations available," he said.

Dr. Kumar's presentation covered the landscape of $\mathrm{MM}$, from solitary bone lesions to smoldering disease to

Presented by Shaji K. Kumar, MD, Division of Hematology, Mayo Clinic Cancer Center, Rochester, Minnesota.

Dr. Kumar has disclosed that he has received grant/research support from Abbott Laboratories, Amgen Inc., Celgene Corporation, Janssen Pharmaceutica Products, LP, Novartis Pharmaceuticals Corporation, sanofi-aventis U.S., and Takeda Pharmaceuticals North America, Inc.; he has served as a scientific advisor for Amgen Inc., Bristol-Myers Squibb Company, Celgene Corporation, GlycoMimetics, Janssen Pharmaceutica Products, LP, sanofi-aventis U.S., and Takeda Pharmaceuticals North America, Inc.; and he has received consulting fees/honoraria from Kesios Therapeutics, NOXXON Pharma AG, and SkylineDx.

Correspondence: Shaji K. Kumar, MD, Division of Hematology, Mayo Clinic Cancer Center, 200 First Street SW, Rochester, MN 55905.

Email: kumar.shaji@mayo.edu the primary treatment of active myeloma and relapsed disease.

\section{Solitary Plasmacytoma}

Solitary plasmacytomas-biopsy-proven solitary lesions of bone or soft tissue, with evidence of clonal plasma cells_can exist with normal bone marrow or with $<10 \%$ clonal bone marrow plasma cells. The NCCN Guidelines have made the recommendations for treating solitary plasmacytomas more specific, highlighting the need to administer adequate doses of radiation therapy-specifically at least 40 to 50 Gy to the involved field - with or without surgery. Disappearance of the M protein is a good prognostic indicator for reduced risk of progression. Follow-up with imaging is important to monitor for new lesions.

\section{Smoldering Myeloma}

At 5 years, approximately $50 \%$ of patients diagnosed with smoldering MM will develop overt disease. These patients represent a mix of those with monoclonal gammopathy of unknown significance, who are less likely to experience disease progression, and patients with early active disease. "There's no foolproof way of saying which patients are going to progress to myeloma and which are not, but we have made significant progress in risk-stratification models," Dr. Kumar said. 
Updates to Multiple Myeloma Management

Risk stratification is important because it can inform the approach to these patients. Patients with 3 risk factors have a 4-fold increased risk of progression compared with those with only 1 risk factor. "If the risk is so high, it makes sense not to allow something catastrophic to happen to the patient, such as renal failure or spinal cord compression," he remarked.

There is, indeed, evidence that early intervention for patients with high-risk smoldering MM has a payoff. The Quiridex trial ${ }^{2}$ found that treatment with lenalidomide/dexamethasone (Rd) induction, followed by lenalidomide maintenance, improved both progression-free survival (PFS; hazard ratio [HR], 0.24; $P<.0001$ ) and overall survival (OS; HR, $0.41 ; P=.024)$.

"We have been able to alter the natural history of this disease and improve OS of patients with high-risk smoldering MM. This is a paradigm shift for how we think about myeloma," Dr. Kumar said. The current NCCN recommendation continues to be observation for patients with smoldering MM. Although it may seem appropriate to treat those at high risk of progression, the guidelines currently recommend treating high-risk patients only within a clinical trial. Some of these studies are asking whether patients with somewhat less risk can also benefit from early treatment.

\section{Newly Diagnosed Patients}

The key issue with newly diagnosed patients remains their eligibility for autologous stem cell transplant (ASCT). Transplant-eligible patients receive induction therapy, consolidation, and transplant, followed by maintenance therapy. Patients who are transplant-ineligible should continue their primary myeloma regimen, with or without maintenance.

Risk stratification (based on the International Staging System [ISS] and cytogenetic testing) is important. Although the results of these tests will not necessarily change initial treatment, they are informative for prognosis and can help select high-risk patients for whom more aggressive therapy may be beneficial, he said.

\section{Primary Therapy for Transplant Candidates}

For patients eligible for ASCT, NCCN's preferred regimen is bortezomib/lenalidomide/dexamethasone
(VRd; category 1) or bortezomib/cyclophosphamide/dexamethasone (VCd). Other recommended therapies include bortezomib/doxorubicin/dexamethasone (category 1), carfilzomib/lenalidomide/ dexamethasone, and ixazomib/lenalidomide/dexamethasone (category 2B).

Regimens considered useful in certain circumstances include bortezomib/dexamethasone (category 1), bortezomib/thalidomide/dexamethasone (category 1), Rd (category 1), and dexamethasone/ thalidomide/cisplatin/doxorubicin/cyclophosphamide/etoposide/bortezomib (VTD-PACE).

SWOG S0777 was the first prospective randomized phase III trial to directly compare VRd to Rd in those with newly diagnosed MM. ${ }^{3}$ This trial enrolled 471 patients with untreated myeloma meeting CRAB (hypercalcemia, renal insufficiency, anemia, bone disease) criteria and randomized them to VRd or Rd, the standard at the time. Median PFS was 43 months with VRd versus 30 in the $R d$ arm $(P=.0037)$, which translated into an OS benefit, with median survivals of 75 and 64 months, respectively $(P=.025)$. Although the complete response rate nearly doubled with the triplet $(15.7 \%$ vs $8.4 \%)$, overall response rates were not different between the arms.

SWOG S0777 provided compelling evidence that the triplet VRd is more effective than $\mathrm{Rd}$ in the frontline setting for patients with MM. "Based on this trial, VRd has become the standard initial therapy in newly diagnosed patients outside of a clinical trial," Dr. Kumar indicated. Other induction regimens with proven efficacy include the triplet of thalidomide/lenalidomide/dexamethasone (VTd) and VCd. In today's practice, these 3 regimens can be considered, but VRd is the preferred regimen based on its OS advantage, Dr. Kumar said.

\section{Role of Stem Cell Transplant}

The best data in support of early ASCT with current drugs are from the French IFM 2009 trial in which patients were randomized to immediate ASCT after 3 cycles of VRd (followed by ASCT consolidation and lenalidomide maintenance) or to 8 cycles of VRd then maintenance, with ASCT at relapse. ${ }^{4} \mathrm{Re}$ sults of this trial showed clear superiority of upfront ASCT as measured by PFS (50 vs 36 months; HR, $0.65 ; P<.001)$, higher complete response rates $(59 \%$ 
vs $48 \%$ ), and high rates of minimal residual disease negativity (79\% vs 65\%).

"But the data also supports continuing on the same therapy and delaying transplant if desired by the patient," Dr. Kumar said. "That's supported by the fact that there was no difference in OS at 4 years between early ( $81 \%$ ) versus delayed ASCT (82\%)."

\section{What To Do After Transplant?}

Optimal therapy post-ASCT is still not completely clear, except for the role of maintenance therapy. A recent meta-analysis of 3 large randomized controlled trials of lenalidomide maintenance after ASCT involving 1,208 patients found median PFS to be 52.8 months for maintenance and 23.5 months for observation (HR, 0.48; 95\% CI, 0.14-0.55). ${ }^{5}$ Median OS was not reached by 79.5 months in the maintenance arm, but was 86 months in the control arm (HR, 0.75; P=.001).

Although most subgroups derived benefit from lenalidomide, patients with ISS stage III and highrisk cytogenetics did not. For these patients, current practice is to use bortezomib for maintenance, rather than lenalidomide. Bortezomib's utility as maintenance in the post-ASCT setting was shown for the first time in the phase III HOVON group trial, which found a striking benefit for bortezomib maintenance in patients with $17 \mathrm{p}$ deletions. "This formed the basis for NCCN's recommendation to use bortezomib for high-risk disease," Dr. Kumar commented.

Also for high-risk disease, tandem ASCT has shown efficacy over single transplant, as demonstrated in a retrospective study by Cavo et $\mathrm{al}^{7}$ in which patients with $17 \mathrm{p}$ deletions with or without $\mathrm{t}(4: 14)$ translocations experienced better survival at 4 years, $76 \%$ versus $33 \%$ ( $\mathrm{HR}, 0.22 ; \mathrm{P}<.001)$, with tandem ASCT. More recently, the large European EMN02/ H095 trial also evaluated tandem transplant and found it improved PFS, especially among patients with high-risk cytogenetics (HR, 0.66; $P=.030) .{ }^{8}$

Despite these supportive data for tandem transplant, "nothing in MM is that simple," Dr. Kumar remarked.

The large US transplant trial, BMT CTN 0702 (StaMINA), yielded different findings, showing no benefit for double transplant compared with single transplant followed by lenalidomide maintenance for the treatment of newly diagnosed patients. ${ }^{9}$ Despite the discordance, and the need for longer follow-up, Dr. Kumar suggested that it is reasonable, after initial transplant, to discuss tandem transplant in patients with $17 \mathrm{p}$ deletions.

\section{Patients Not Eligible for Transplant}

The NCCN Guidelines provide recommendations for primary therapy in nontransplant candidates. Preferred regimens include VRd (category 1), lenalidomide/low-dose dexamethasone (category 1), and VCd; other recommended regimens include carfilzomib/lenalidomide/dexamethasone, carfilzomib/cyclophosphamide/dexamethasone, and ixazomib/lenalidomide/dexamethasone. Bortezomib/dexamethasone is considered useful in certain circumstances.

For maintenance, the preferred regimen is lenalidomide (category 1), based on results of the FIRST tri$\mathrm{al},{ }^{10}$ which established that $\mathrm{Rd}$ (continuous or for 18 months) improved not only PFS but also OS.

\section{Relapsed MM}

"Patients with MM invariably experience relapse, and this is one place [in the guideline] where we have lots of choices, based on multiple phase III trials in relapsed disease that have provided clarity," he said. Choices include combinations of next-generation proteasome inhibitors, including the oral drug ixazomib, along with monoclonal antibodies and more intensive combinations for certain circumstances, such as aggressive, rapidly proliferating disease.

Multiple different regimens can be considered for patients who have undergone previous therapies prior to relapse. The NCCN Guidelines currently list 8 preferred options for these patients. Further, the guidelines list 21 other recommended therapies, which include doublets and triplets using bendamustine, bortezomib, carfilzomib, cyclophosphamide, daratumumab both as a monotherapy and in combination, pomalidomide, elotuzumab, ixazomib, lenalidomide, and panobinostat. Regimens listed as useful in certain circumstances include bendamustine monotherapy, high-dose cyclophosphamide, dexamethasone/cyclophosphamide/etoposide/cisplatin (DCEP), and dexamethasone/thalidomide/ cisplatin/doxorubicin/cyclophosphamide/etoposide (DT-PACE) with or without bortezomib. 
Updates to Multiple Myeloma Management

According to Dr. Kumar, the general principles of treating relapsed disease are (1) biochemical relapse does not always warrant the start of a new therapy; (2) duration of initial response defines disease biology; (3) triplets ( 2 active classes + dexamethasone) are preferred over doublets, and at least 1 drug should be included from a nonrefractory class; (4) performance status, age, and comorbidities should be considered when selecting therapy; (5) prior and residual toxicities should be taken into account; and (6) patients should be treated until they have experienced maximum response and should be maintained on one drug until progressive disease or intolerability.

For patients with relapsed disease not refractory to bortezomib, 3 phase III trials evaluating new agents in combination with bortezomib/dexamethasone have shown improvements (or trends) in PFS: ENDEAVOR (carfilzomib), ${ }^{11}$ PANORAMA 1 (panobinostat), ${ }^{12}$ CASTOR (daratumumab), ${ }^{13}$ as well as a randomized phase II study of elotuzumab. ${ }^{14}$
For patients not refractory to lenalidomide, 4 phase III trials have evaluated drugs in combination with Rd: ASPIRE (carfilzomib), ${ }^{15}$ TOURMALINEMM1 (ixazomib), ${ }^{16}$ POLLUX (daratumumab), ${ }^{17}$ and ELOQUENT-2 (elotuzumab). ${ }^{18}$ These trials similarly reported improved PFS, and ASPIRE and ELOQUENT also reported improved OS. "This highlights the fact that patients with relapsed disease should ideally be treated with at least 2 different classes of drugs," he said.

For patients not refractory to either lenalidomide or bortezomib - who are much like newly diagnosed patients-options include a second use of the patient's initial induction therapy. The more common group, however, are patients who become refractory to both of these standard agents, and need to be exposed to a new class of drugs. A second ASCT at relapse can improve PFS, especially in those who experienced a relatively long disease-free survival interval after the first ASCT. Older (previously unused) agents can also produce durable responses in this setting.

\section{References}

1. Dispenzieri A, Kyle RA, Katzmann JA, et al. Immunoglobulin free light chain ratio is an independent risk factor for progression of smoldering (asymptomatic) multiple myeloma. Blood 2008;111:785-789.

2. Mateos MV, Hernández MT, Giraldo $P$, et al. Lenalidomide plus dexamethasone for high-risk smoldering multiple myeloma. N Engl J Med 2013;369:438-447.

3. Durie BG, Hoering A, Abidi MH, et al. Bortezomib with lenalidomide and dexamethasone versus lenalidomide and dexamethasone alone in patients with newly diagnosed myeloma without intent for immediate autologous stem-cell transplant (SWOG S0777): a randomised, open-label, phase 3 trial. Lancet 2016;389:519-527.

4. Attal M, Lauwers-Cances V, Hulin C, et al. Lenalidomide, bortezomib, and dexamethasone with transplantation for myeloma. N Engl J Med 2017;376:1311-1320.

5. McCarthy PL, Holstein SA, Petrucci MT, et al. Lenalidomide maintenance after autologous stem-cell transplantation in newly diagnosed multiple myeloma: a meta-analysis. J Clin Oncol 2017;35:3279-3289.

6. Sonneveld P, Schmidt-Wolf I, van der Holt B, et al. HOVON-65/GMMGHD4 randomized phase III trial comparing bortezomib, doxorubicin, dexamethasone (PAD) vs VAD followed by high-dose melphalan (HDM) and maintenance with bortezomib or thalidomide in patients with newly diagnosed multiple myeloma (MM) [abstract]. Blood 2010;116:Abstract 40.

7. Cavo M, Salwender H, Rosinol L, et al. Double vs single autologous stem cell transplantation after bortezomib-based induction regimens for multiple myeloma: an integrated analysis of patient-level data from phase III European studies [abstract]. Blood 2013;122:Abstract 767.

8. Cavo M, Palumbo A, Zweegman S, et al. Upfront autologous stem cell transplantation (ASCT) versus novel agent-based therapy for multiple myeloma (MM): a randomized phase 3 study of the European Myeloma Network (EMN02/HO95 MM trial) [abstract]. J Clin Oncol 2016;34(Suppl):Abstract 8000 .

9. Stadtmauer EA, Pasquini MC, Blackwell C, et al. Comparison of autologous hematopoietic cell transplant (autoHCT), bortezomib, lenalidomide (Len) and dexamethasone (RVD) consolidation with lenalidomide maintenance
(ACM), tandem autoHCT with lenalidomide maintenance (TAM) and autoHCT with lenalidomide maintenance (AM) for up-front treatment of patients with multiple myeloma (MM): primary results from the randomized phase III trial of the Blood and Marrow Transplant Clinical Trials Network (BMT CTN 0702-StaMINA trial) [abstract]. Presented at the 2016 ASH Annual Meeting; December 1-4, 2016; San Diego, California. Abstract LBA-1.

10. Benboubker L, Dimopoulos MA, Dispenzieri A, et al. Lenalidomide and dexamethasone in transplant-ineligible patients with myeloma. N Engl J Med 2014:371:906-917.

11. Dimopoulos, MA, Moreau $\mathrm{P}$, Palumbo A, et al. Carfilzomib and dexamethasone versus bortezomib and dexamethasone for patients with relapsed or refractory multiple myeloma (ENDEAVOR): a randomised, phase 3, open-label, multicentre study. Lancet Oncol 2016;17:27-38.

12. San-Miguel JF, Hungria VT, Yoon SS, et al. Panobinostat plus bortezomib and dexamethasone versus placebo plus bortezomib and dexamethasone in patients with relapsed or relapsed and refractory multiple myeloma: a multicentre, randomised, double-blind phase 3 trial. Lancet Oncol 2014;15:1195-1206.

13. Palumbo A, Chanan-Khan A, Weisel K, et al. Daratumumab, bortezomib, and dexamethasone for multiple myeloma. N Engl J Med 2016;375:754766.

14. Jakubowiak A, Offidani M, Pegourie B, et al. Randomized phase 2 study: elotuzumab plus bortezomib/dexamethasone vs bortezomib/dexamethasone for relapsed/refractory MM. Blood 2016;127:2833-2840.

15. Stewart AK, Rajkumar SV, Dimopoulos MA, et al. Carfilzomib, lenalidomide, and dexamethasone for relapsed multiple myeloma. N Engl J Med 2015;372:142-152.

16. Moreau P, Masszi T, Grzasko N, et al. Oral ixazomib, lenalidomide, and dexamethasone for multiple myeloma. N Engl J Med 2016;374:1621-1634.

17. Dimopoulos MA, Oriol A, Nahi H, et al. Daratumumab, lenalidomide, and dexamethasone for multiple myeloma. N Engl J Med 2016;375:1319-1331.

18. Lonial S, Dimopoulos M, Palumbo A, et al. Elotuzumab therapy for relapsed or refractory multiple myeloma. N Engl J Med 2015;373:621-631. 